\title{
Absolute and relative reliability of several measures of static postural stability calculated using a GYKO inertial sensor system
}

\author{
JANUSZ JAWORSKI, TADEUSZ AMBROŻY, GRZEGORZ LECH, MiCHAŁ SPIESZNY, \\ PRZEMYSŁAW BUJAS, MICHAŁ ŻAK, WIESŁAW CHWAŁA*
}

Department of Physical Education and Sport, University of Physical Education in Krakow, Kraków, Poland.

\begin{abstract}
Purpose: The aim of this study was to analyse absolute and relative reliability of a number of postural static stability measures obtained from a GYKO inertial sensor system in young adults. Methods: The study examined 29 healthy non-athlete young adults. A test was performed for $30 \mathrm{~s}$ while standing on one foot, without moving, with eyes open and arms relaxed along the sides of the body. The examinations were performed twice, with a one-week interval. Relative reliability was measured using the intraclass correlation coefficient (ICC) and the 95\% confidence interval (95\% CI), whereas the absolute reliability was evaluated based on the standard error of measurement (SEM) and the minimal detectable change (MDC). Results: The results of this study showed moderate to good relative reliability scores for all the postural stability measures, with ICC values ranging from 0.62 to 0.70 . For most of the analysed variables, SEM\% ranged from ca. 10 to $14 \%$. Relatively high SEM\% values were obtained only for two variables (Area, Convex Hull Area). Conclusions: The low costs of GYKO inertial sensor systems, the fast and easy installation, the mobility and high reliability of the measurement of postural stability show that it can be effective alternative to stabilographic platforms.
\end{abstract}

Key words: balance, postural stability, accelerometer, test-retest reliability, intraclass correlation coefficient

\section{Introduction}

Postural stability is considered an important component that determines the effectiveness of human motor activity. Postural stability is needed in many sports, both during recruitment and when monitoring the effects of training [28]. An adequate level of postural stability determines the safe and independent performance of everyday activities and reduces the risk of falls, especially in older people [17].

Balance has been defined as the ability to maintain the centre of gravity inside the support area marked by the external contours of the feet [26]. Maintaining balance is controlled by the central nervous system by means of input information from the vestibular, visual, tactile and proprioceptive systems. The informa- tion obtained is processed by the central nervous system and then transferred to the effector organs [12].

Currently, there is no uniform method to measure static balance. The most popular technique used for evaluation of postural stability is measurement of position and displacements of the centre of gravity by means of force platforms ("golden standards") [17]. Biodex Balance System SD is also often used to evaluate postural stability [25]. There are many possible types of balance testing protocols: with arms crossed on the chest, on the hips, or relaxed at the sides of the body; standing on one leg or both legs; with eyes open or closed; on the stable or unstable ground [25]. Balance platforms are usually very big and expensive, and their installation and software are complex [18], all of which substantially limits their application. For these reasons, postural stability has been evaluated for

\footnotetext{
* Corresponding author: Wiesław Chwała, Institute of Biomedical Sciences, University of Physical Education in Krakow, al. Jana Pawła II 78, 31-571 Kraków, Poland. Phone: 504303896, e-mail: wieslaw.chwala@awf.krakow.pl

Received: March 15th, 2020

Accepted for publication: April 13th, 2020
} 
many years [13], [22] with the use of various accelerometers because they are cheaper, more mobile, easy to use, and based on advanced wireless data transmission technologies [17], [16]. ICC reliability results during postural stability measurements performed by means of accelerometers are good, usually over 0.75 [18], [19], [27]. Some studies have also demonstrated a strong correlation between results obtained from force platforms and accelerometer data.

The location of accelerometers on human body is also an important issue due to links between the movements of individual body segments and the COM (centre of mass) displacements used to maintain postural stability. Accelerometers are often placed on the lumbar section of the spine (L3), which is the approximate height of the COM [27]. Furthermore, based on correlation coefficients, Golema [10] determined the relationships between COM and body trunk displacements. The results demonstrated unequivocally that body trunk displacements (displacements of a point located near the centre of body trunk gravity) can be considered the same as those of COM. This assumption applies only to maintaining postural stability in undisturbed conditions. Therefore, in our study, the Gyko inertial sensor system was attached to the body trunk of participants at the height of T1-T2, as recommended by the manufacturer. There are no published studies (to the authors' knowledge) that have analysed the reliability of the measures of static stability obtained through an inertial system placed in the upper back.

The main aim of this study was to use the GYKO accelerometer (Microgate, Bolzano, Italy) to evaluate the measurement reproducibility (ICCs) of selected variables that characterize postural stability.

\section{Methods}

\subsection{Participants}

The study examined 29 healthy non-athlete students. In the last 3 months, the subjects did not report any serious injuries such as ankle or knee joint injury, chronic ligament dislocation or other injuries to the lower limb. They also did not report neurological problems related to balance disorders. All the tests were conducted in 2017. The tests and anthropometric measurements were performed in accordance with the Declaration of Helsinki. The examinations were approved by the Bioethics Committee at the Regional Medical Chamber in Kraków, Poland (approval No. 159/KBL/OIL/2017). The tests were conducted within statutory research No. 106/BS/IS/2016. Statistical characteristics of basic somatic parameters and age of the study participants are presented in Table 1.

Table 1. Statistical characteristics of basic somatic parameters and age of the study participants

\begin{tabular}{|l|c|c|c|}
\hline \multicolumn{1}{|c|}{ Variable } & $\bar{x}$ & SD & $V[\%]$ \\
\hline Body height $[\mathrm{cm}]$ & 179.82 & 6.87 & 3.82 \\
\hline Body mass $[\mathrm{kg}]$ & 75.55 & 7.62 & 10.08 \\
\hline BMI $\left[\mathrm{kg} / \mathrm{m}^{2}\right]$ & 23.69 & 2.08 & 8.78 \\
\hline GYKO height $[\mathrm{cm}]$ & 148.13 & 7.21 & 4.86 \\
\hline Chronological age [years] & 21.67 & 0.88 & 6.90 \\
\hline
\end{tabular}

\subsection{Testing protocol}

The participants performed all the tests barefoot, in the same room, between 8 a.m. and 3 p.m. A test was performed for $30 \mathrm{~s}$ while standing on one foot, without moving, with eyes open and arms relaxed along the sides of the body. The group of university students always performed the first and second series (one test each time) of examinations at the same time of the day. Both test series were supervised by the same research team. The tests and retests were conducted with a one-week interval (no trial test was performed before). This approach has been often used in studies examining test reliability [24]. During the interval between the test and retest, the students did not practise any sport and did not take up any additional physical activity. During the first examination, we determined the height at which the GYKO system was to be attached. According to the manufacturer's recommendations, this height should be set at the level of the T1-T2 thoracic vertebrae (determined by palpation based on spinous processes). During the second examination, the GYKO system was attached again at the same height. The height of GYKO was measured using a Martina anthropometer. The tension of the GYKO attaching straps (chest circumference) was also adjusted to each student. With the latest generation of the components, the system allows for the measurement of accelerations of up to $16 \mathrm{~g}$ and angular velocities of up to $2000 \% \mathrm{~s}$ with an acquisition frequency of $1000 \mathrm{~Hz}$. The wireless transmission protocol was used to transfer data recorded by the GYKO inertial sensor to the laptop (Lenovo Yoga 500-15 i5-6200/8GB/1000/Win10). Furthermore, the technical specifications of the GYKO inertial system are available from the website [29]. 


\subsection{Static stability measures}

The focus of the analysis was on the following variables that characterize postural stability: Area $\left[\mathrm{mm}^{2}\right]$, Area Convex Hull $\left[\mathrm{mm}^{2}\right]$, Length [mm], Length (AP) [mm], Length (ML) [mm], Mean Velocity [mm/s], Mean Velocity (AP) $[\mathrm{mm} / \mathrm{s}]$, Mean Velocity (ML) $[\mathrm{mm} / \mathrm{s}]$. A detailed description of all the analysed variables is presented in the User Manual, Version1.1.1.9, pages 127-129 [29], [30].

\subsection{Statistical analysis}

1. The Shapiro-Wilk test was used to test variables for normal distribution, whereas Levene's test was employed to assess the equality of variances.

2. Basic statistical parameters were computed for all the variables in both series of examinations (test, retest) and for the material in total.

3. The relative reliability of the repeated test [2] was evaluated using the intraclass correlation coefficient (ICC) with 95\% confidence interval $(95 \%$ CI). For this purpose, we computed ICCs between test and retest results. For variables whose distribution was not normal, raw results were logarithmized to fit the normal distribution, then correlation coefficients were calculated for these values.

4. Apart from ICCs, we also computed standard error of measurement (SEM), which is used to express absolute measurement reliability [2], according to the formula:

$$
\mathrm{SEM}=\mathrm{SD} \sqrt{1-\mathrm{ICC}},
$$

where:

SEM - standard error of measurement,

SD - standard deviation from the test 1 and 2,

ICC - intraclass correlation coefficient.

5. Next, SEM\% values were computed as

$$
\mathrm{SEM} \%=(\mathrm{SEM} / \text { grand mean }) \times 100 .
$$

This method was used to express SEM as a percentage of the mean value from the test and retest for each variable to allow comparison of absolute reliability between the measurements obtained in various units [5].

6. SEM was used to evaluate the minimal detectable change (MDC), interpreted as the lowest value of changes needed to exceed the error of measurement for two repeated tests at specific CI. Computations were performed for $95 \% \mathrm{CI}$ using the formula:

$$
\mathrm{MDC}_{95}=\mathrm{SEM} \times 1.96 \times \sqrt{2} .
$$

7. Next, $\mathrm{MDC}_{95}$ was expressed as the percentage of the mean from the test and retest, according to the formula:

$$
\mathrm{MDC}_{95 \%}=(\mathrm{MDC} 95 / \text { grand mean }) \times 100 \text {. }
$$

8. The differences between means from the test and retest used the repeated-measures Student's $t$-test for dependent samples. We used the non-parametric Wilcoxon pair test if the distribution of variables differed from normal. Furthermore, the Cohen effect was also computed for each variable [3].

The calculations were performed using the STATISTICA 13.1 PL for Windows software package and an Excel spreadsheet. Furthermore, the effect size was determined by means of GPower 3.1 freeware,

\begin{tabular}{|c|c|c|c|c|c|c|c|}
\hline \multirow{2}{*}{ Variable [unit of measurement] } & \multicolumn{2}{|c|}{ Test } & \multicolumn{2}{|c|}{ Retest } & \multirow{2}{*}{$t, W$} & \multirow{2}{*}{$p$} & \multirow{2}{*}{$C^{d}$} \\
\hline & $\bar{x}$ & SD & $\bar{x}$ & SD & & & \\
\hline Area $\left[\mathrm{mm}^{2}\right]$ & 5055.87 & 3167.88 & 4315.36 & 3133.59 & $1.56^{\mathrm{W}}$ & 0.11 & 0.23 \\
\hline Convex Hull Area $\left[\mathrm{mm}^{2}\right]$ & 3971.22 & 2376.74 & 3640.49 & 2722.94 & $0.85^{\mathrm{W}}$ & 0.39 & 0.13 \\
\hline Length $[\mathrm{mm}]$ & 933.82 & 218.97 & 981.66 & 271.35 & -1.30 & 0.20 & 0.14 \\
\hline Length (AP) [mm] & 612.55 & 133.73 & 626.61 & 141.86 & -0.66 & 0.51 & 0.07 \\
\hline Length (ML) [mm] & 573.09 & 161.08 & 617.67 & 229.58 & -1.43 & 0.16 & 0.16 \\
\hline Mean Velocity $[\mathrm{mm} / \mathrm{s}]$ & 30.85 & 6.24 & 32.43 & 6.96 & -1.30 & 0.20 & 0.17 \\
\hline Mean Velocity (AP) [mm/s] & 20.24 & 4.42 & 20.70 & 4.68 & -0.65 & 0.51 & 0.07 \\
\hline Mean Velocity (ML) [mm/s] & 18.93 & 5.32 & 20.40 & 3.58 & -0.85 & 0.39 & 0.24 \\
\hline
\end{tabular}
which is widely used in social studies [8].

Table 2. Basic statistic characteristics of the analysed postural stability indices with evaluation of the significance of differences in test-retest results and Cohen's effect size

Without marking - the value of Student- $t$ statistic for dependent samples; $W$ - value of the Wilcoxon test statistic; $C-$ Cohen's effect size; $d>0.2=$ small, $>0.5=$ medium, $>0.8=$ large .

* Significant at $p \leq 0.05$. 


\section{Results}

In Table 2, basic statistical characteristics for test and retest variables are presented. The table also contains the evaluation of the significance of differences in the results of individual variables from the first and the second measurement and Cohen's effect sizes. No statistically significant differences were found between the results of the first and the second measurement. According to the classification proposed by Cohen, the effect size for 6 variables should be considered small (values of statistics below 0.2). Mean effect was observed only for two variables (Area, Mean Velocity (ML)). However, in both cases, Cohen statistics significantly exceeded boundary values of 0.20 .

Based on the results of the first and the second measurements, we computed the reliability indices of the analysed variables that characterize postural stability. The values of intraclass correlation coefficients (ICC), standard error of measurement (SEM), and minimal detectable change $\left(\mathrm{MDC}_{95}\right)$ are presented in Table 3. The relative reliability of the repeated test for all analysed variables of ICC was in the very narrow range of 0.62 to 0.70 . The lowest ICCs were obtained for the ellipse surface area which contains $95 \%$ of the points of a specific trajectory (Area). Furthermore, the highest ICCs were obtained for Mean Velocity and Length.

In Table 3, also standard error of measurement (SEM), which is used to express absolute measurement reliability, is presented. In general, for most of the analysed variables, SEM\% ranged from ca. 10 to $14 \%$. Relatively high SEM\% values were obtained for only two variables (Area, Convex Hull Area). It should be emphasized that despite the relatively high value of SEM \% for both variables, high ICCs of 0.62 and 0.67 were found, respectively.
Using the SEM values, we calculated the minimal detectable changes, $\mathrm{MDC}_{95}$ and $\mathrm{MDC}_{95 \%}$. The most analysed variables (Length, Length (ML), Length (AP), Mean Velocity, Mean Velocity (ML), Mean Velocity (AP)) were contained in a narrow range from 29 to $38 \%$. The highest values of $\mathrm{MDC}_{95 \%}$ were observed for Area and Convex Hull Area, with 114 and 106\%, respectively.

\section{Discussion}

The main aim of this study was to evaluate the reliability of measurements of selected variables that characterize postural stability. The Microgate GYKO inertial sensor system was designed for the purpose. All equipment used in medical and sports diagnosis should be characterized by high measurement reliability. The reliability and agreement of measurements is an important criterion that ensures high-quality examinations [15], [14]. Reliability has been defined as "the ratio of variability between subjects (e.g., patients) or objects (e.g., computed tomography scans) to the total variability of all measurements in the sample" [15]. Ten models were described in the literature to compute ICCs [21]. Since each model is based on different assumptions and leads to different interpretations, researchers should clearly define the ICC form they use in their computations. In our study, the credibility of postural stability results was evaluated by computation of intraclass correlation coefficients (ICCs [26], [17]) and standard measurement error (SEM). ICC was computed by means of a two-way model of random effects with absolute agreement and single measurement [21]. An important factor in the determination of measurement reliability is also the time between test

Table 3. The values of intraclass correlation coefficients (ICC), standard error of measurement (SEM) and minimal detectable change $\left(\mathrm{MDC}_{95}\right)$ for the analysed indices of postural stability

\begin{tabular}{|l|c|c|c|c|c|c|}
\hline \multicolumn{1}{|c|}{ Variable [unit of measurement] } & ICC & ICC $(95 \% \mathrm{CI})$ & SEM & SEM $\%$ & MDC $_{95}$ & MDC $_{95 \%}$ \\
\hline Area $\left[\mathrm{mm}^{2}\right]^{*}$ & 0.62 & $0.33 \div 0.80$ & 1938.87 & 41.13 & 5372.60 & 114.66 \\
\hline Convex Hull Area $\left[\mathrm{mm}^{2}\right]^{*}$ & 0.67 & $0.40 \div 0.83$ & 1458.36 & 38.31 & 4042.36 & 106.21 \\
\hline Length [mm] & 0.70 & $0.45 \div 0.85$ & 134.50 & 14.04 & 372.81 & 38.92 \\
\hline Length (AP) [mm] & 0.65 & $0.37 \div 0.82$ & 79.77 & 12.87 & 221.13 & 35.68 \\
\hline Length (ML) [mm] & 0.69 & $0.43 \div 0.84$ & 76.17 & 12.75 & 211.13 & 35.46 \\
\hline Mean Velocity [mm/s] & 0.70 & $0.45 \div 0.85$ & 3.34 & 10.57 & 9.25 & 29.23 \\
\hline Mean Velocity (AP) $[\mathrm{mm} / \mathrm{s}]$ & 0.66 & $0.39 \div 0.83$ & 2.63 & 12.84 & 7.28 & 35.10 \\
\hline Mean Velocity (ML) $[\mathrm{mm} / \mathrm{s}]$ & 0.68 & $0.42 \div 0.84$ & 2.51 & 12.76 & 6.96 & 35.38 \\
\hline
\end{tabular}

ICC - coefficient of intraclass correlation, 95\% CI - confidence interval, SEM - standard error of measurement, SEM $\%$ - percentage standard error of measurement, MDC - minimal detectable change, ${ }^{*}$ - Pearson correlation coefficient was computed based on the logarithmized values. 
and retest. Park et al. [24] analysed reports from 2000-2017 by searching the MEDLINE, Embase, CINAHL, and PsycINFO databases. Of $12.641 \mathrm{ex}-$ aminations, 95 studies were qualified for further analysis. The detailed analysis showed that the median of the time range for test-retest reliability was 14 days. The researchers analysed an additional 17 studies with high reliability indices and found that the mean interval between test and retest was 12.88 days. In our study, this time was 7 days, therefore it was within the proposed interval range between two tests. Huang et al. [11] also indicated that the interval between test and retest should be 1-2 weeks.

Another important issue during determination of measurement reliability is the adoption of unreliable criteria. The related literature suggests a number of suggestions for ranges of reliability and their interpretation [1], [5]-[7]. In our study, we used the proposal by Fleiss et al.[9], who interpreted ICCs in the following manner: $<0.4=$ low reliability; $0.4-0.75=$ moderate to good reliability; and $>0.75=$ excellent reliability. With regards to postural stability, there are many scientific papers on its measurement reliability. The results of reliability measurements are within a very broad range [4] due to the various methodological approaches and other factors: varying test durations, different testing positions, stable or unstable ground, tests performed with eyes open or closed, tests performed on healthy individuals or those with geriatric problems, etc. The obtained ICCs values for variables concerning postural stability in our study ranged from 0.62 to 0.71 . According to the adopted classification by Fleiss et al. [9], these results should be regarded as high. They were slightly worse than those obtained using the YEI 3-Space Sensor Wireless accelerometer, for which ICCs ranged from 0.736 to 0.972 [27]. However, in this case, the examinations were performed when the subject was standing on both feet.

Many authors explain that apart from the interpretation of ICCs, it is important in practice that absolute values of measurement reliability are also evaluated [1], [5]. This methodical approach was also used in the present study by computing SEM\% and $\mathrm{MDC} \%$. As emphasized by Atkinson and Nevill [2], the higher the SEM \%, the lower the absolute test reliability and the lower the precision of the obtained results. In our study, SEM\% for Length [mm], Length (AP) [mm], Length (ML) $[\mathrm{mm}]$, Mean Velocity $[\mathrm{mm} / \mathrm{s}]$, Mean Velocity (AP) $[\mathrm{mm} / \mathrm{s}]$ and Mean Velocity (ML) $[\mathrm{mm} / \mathrm{s}]$ ranged from ca. 9 to $14 \%$. This demonstrates very good absolute measurement reliability. Only for Area $\left[\mathrm{mm}^{2}\right]$ and Convex Hull Area $\left[\mathrm{mm}^{2}\right]$, SEM\% had significantly higher values (around 40\%). Furthermore, SEM values were used for the computation of minimal detectable change (MDC\%). This index is defined as the minimal value of changes needed to distinguish the actual change from the change caused by the variability of results or measurement errors [23]. In our study, we obtained a relatively high MDC\% for Area $\left[\mathrm{mm}^{2}\right]$ and Convex Hull Area $\left[\mathrm{mm}^{2}\right]$. These results, however, are not surprising due to the method of SEM computation. Relatively high values of measurement error for both variables also led to high $\mathrm{MDC} \%$ values. For other analysed variables, MDC\% values were relatively average and ranged from $26 \%$ to $38 \%$.

\section{Limitation of the study}

The main benefit of this study is the use of a modern 3-axis accelerometer for postural stability analysis, using the latest wireless data transmission technologies. However, the authors are aware of the limitations of this report. Therefore, in future research, we propose:

- to increase the size of the research group,

- to carry out the tests in different chronological age groups of both sexes,

- to carry out the tests in different positions,

- attaching the GYKO device in the lumbar region of the spine (L3),

- the results obtained from the GYKO accelerometer should be compared with data from the force platform.

Despite the limitations presented above, the GYKO inertial system should be recommended for wide use in clinical trials. A wide practical application of the system is possible due to its great versatility and small dimensions. The device is delivered with easy-to-install software. The results are collected on an ongoing basis and the program records 32 variables characterizing postural stability. All tests can be performed for several variants. High mobility and easiness of use of the equipment make it possible to carry out tests almost anywhere.

\section{Conclusions}

1. All the variables that characterize postural stability indices demonstrated high values of intraclass correlation coefficients (ICCs) between test and retest.

2. The GYKO inertial sensor system can be used as a simple and powerful device for measuring postural stability. 


\section{Data Availability}

The data used to support the findings of this study are available from the corresponding author upon request.

\section{Disclosure statement}

No potential conflict of interest was reported by the authors.

\section{Acknowledgements}

This study is part of the research project „Level of selected morphofunctional indices in badminton players from the Polish national team and untrained men" that was supported by statutory funds from the University of Physical Education in Krakow, Institute of Sport Science (No. 106/BS/IS/2016).

\section{Funding}

Publication was funded under the programme of the Minister of Science and Higher Education "Regional Initiative of Excellence" in the years 2019-2022, Project No. 022/RID/2018/19.

\section{References}

[1] Al-Zahrani E., Gunasekaran C., Callaghan M., GAYDECKI P., BeniteZ D., Oldham J., Within-day and between-days reliability of quadriceps isometric muscle fatigue using mechanomyography on healthy subjects, J. Electromyogr. Kines., 2009, 19 (4). 695-703.

[2] ATKInson G., Nevill A.M., Statistical methods for assessing measurement error (reliability) in variables relevant to sports medicine, Sports Med., 1998, 26 (4), 217-238.

[3] CoHen J., Statistical power analysis for the behavioral sciences, Hillsdale, N.J. Erlbaum, 1998.

[4] Corriveau H., Hébert R., Prince F., Raîche M., Postural control in the elderly: an analysis of test-retest and interrater reliability of the COP-COM variable, Arch. Phys. Med. Rehab., 2001, 82 (1), 80-85.

[5] Da Silva C., De Oliveira Silva D., Briani R., Pazzinatto M., FERRARI D., DE AzEVEDo F., Test-retest reliability of electromyographic signal parameters used to evaluate neuromuscular fatigue in quadriceps femoris muscle, Kines., 2016, 48 (2), 174-181.

[6] Derhon V., Santos R., Brandalize M., Brandalize D., Rossi L., Intra- and inter-examiner reliability in angular measurements of the knee with a smartphone application, Hum. Mov., 2017, 18 (2), 38-43.

[7] Domholdt E., Rehabilitation research: principles and applications, Elsevier, US, 2011, 86-101.

[8] Faul F., ERdfelder E., LANG A.G., Buchner A., G*Power 3: a flexible statistical power analysis program for the social, behavioral, and biomedical sciences, Beh. Res. Met., 2007, 39 (2), 175-191.

[9] Fleiss J.L., LeVIN B., PAIK M.C., Statistical methods for rates and proportions, John Wiley \& Sons, 3rd ed., New York 2003.
[10] Golema M., The size of the displacements of the human body parts maintaining the balance, Studia i Monografie 148, Politechnika Opolska, Opole, 2003.

[11] Huang M., Miller K., Smith K., Fredrickson K., Shilling T., Reliability, validity, and minimal detectable change of balance evaluation systems test and its short versions in older cancer survivors: A pilot study, J. Geriatr. Phys. Ther., 2016, 39 ( 2), 58-63.

[12] Jaffri V., Newman T., Smith B., Miller S., The dynamic leap and balance test (DLBT): a test-retest reliability study, Int. J. Sports Phys. Ther., 2017, 12(4), 512-519.

[13] Kamen G., Patten C., Du C.D., Sison S., An accelerometrybased system for the assessment of balance and postural sway, Gerontol., 1998, 44 (1), 40-45.

[14] KoO T., Li M.A., Guideline of selecting and reporting intraclass correlation coefficients for reliability research, J. Chiropr. Med., 2016, 15 (2), 155-163.

[15] Kottner J., Audigé L., Brorson S., Donner A., Gajewski, HRÓBJARTSSON A., Guidelines for reporting reliability and agreement studies (GRRAS) were proposed, J. Clin. Epidemiol., 2011, 48 (6), 661-671.

[16] Lesinski M., Muehlbauer T., Granacher U., Concurrent validity of the Gyko inertial sensor system for the assessment of vertical jump height in female sub-elite youth soccer players, BMC J. Sport Sci. Med. Reh., 2016, 8, 35.

[17] Lindmark B., Liljenäs Å., Hellström K., Assessment of minor or moderate balance disorders: A reliability study and comparison with healthy subjects, Adv. Physiother., 2012, 14 (1), 3-9.

[18] Mancini M., Salarian A., CARlson-Kuhta P., ZAmpieri C., KING L., CHIARI L., ISway: a sensitive, valid and reliable measure of postural control, J. Neuroeng. Rehabil., 2012, 9(1), 59-66.

[19] Marchetti G.F., Bellanca J., Whitney S.L., Lin J.C., Musolino M.C., Furman G.R., The development of an accelerometer-based measure of human upright static anteriorposterior postural sway under various sensory conditions: test-retest reliability, scoring and preliminary validity of the Balance Accelerometry Measure (BAM), J. Vestibul. Res.Equil., 2013, 23 (4-5), 227-235.

[20] Mathur S., ENG J.J., Macintyre D.L., Reliability of surface EMG during sustained contractions of the quadriceps, J. Electromyogr. Kines., 2005, 15 (1), 102-110.

[21] McGraw K.O., Wong S.P., Forming inferences about some intraclass correlation coefficients, Psychol. Met., 1996, 1, 30-46.

[22] Moe-Nilssen R., Helbostad J.L., Trunk accelerometry as fa measure of balance: control during quiet standing, Gait and Post., 2002, 16 (1), 60-68.

[23] Nair P.M., Hornby G., Behrman A.L., Minimal detectable change for spatial and temporal measurements of gait after incomplete spinal cord injury, Top Spinal Cord. Inj. Rehabil., 2012, 18 (3), 273-281.

[24] Park M., Kang K., Jang S., Lee J., Chang S., Evaluating test-retest reliability in patient-reported outcome measures for older people: A systematic review, Int. J. Nurs. Stud., 2018, 79, 58-69.

[25] Parraca J., Olivares P., Carbonell-Baeza A., Aparicio V., AdSUAR J., GuSI N., Test-retest reliability of Biodex Balance SD on physically active old people, J. Hum. Sport Exerc., 2011, 6 (2), 444-451.

[26] Pollock A.S., Durward B.R., Rowe P.J., Paul J.P., What is balance?, Clin. Rehab., 2000, 14(4), 402-406. 
[27] Saunders N.W., Koutakis P., Kloos A.D., Kegelmeyer D.A., Dicke J.D., Devor S.T., Reliability and validity of a wireless accelerometer for the assessment of postural sway, J. Appl. Biomech., 2015, 31(3), 159-163.

[28] Wrzesień Z., TruszCZYNSKA-BASZAK A., RZEPKA R., Lower extremity muscle strength, postural stability and functional movement screen in female basketball players after ACL re- construction. Preliminary report, Acta Bioeng. Biomech., 2019, 21(2), 71-81.

\section{Internet sources}

[29] http://www.gyko.it/ - Accessed 16 March 2019.

[30] www.gyko.it/Repower/GykoRePower_UserManual_EN.pdf) [accessed: 24 March 2019]. 